\title{
NORTH-HOLLAND
}

\section{HYPERSOLVER: A Graphical Tool for Commonsense Set Theory}

\section{MÜJDAT PAKKAN}

Computer Engineering Department, Boğaziçi University, Bebek, 80815 İstanbul, Turkey

and

VAROL AKMAN*

Department of Computer Engineering and Information Science, Bilkent University, Bilkent, 06533 Ankara, Turkey

Communicated by Frol Gelenbe

\begin{abstract}
This paper investigates an alternative set theory (duc to Aczel) called the Hyperset Theory. Aczel uses a graphical representation for sets and thereby allows the representation of non-well-founded sets. A program, called HYPERSOLVER, which can solve systems of equations defined in terms of sets in the universe of this new theory is presented. This may be a useful tool for cornmonsense reasoning.
\end{abstract}

\section{INTRODUCTION}

Sct theory has long occupied a unique place in mathematics since it allows various other branches of mathematics to be formally defined within it. The theory has ignited many debates on its nature and a number of different axiomatizations were developed to formalize its underlying "philosophical" principles. Collecting entities into an abstraction for further thought (i.e., set construction) is an important process in mathematics, and this brings in assorted problems [5]. The theory had many ground-shaking crises (like

*Corresponding author. Akman's research is supported in part by the Scientific and Technical Research Council of Turkey (TÜBITAK), Grant No. TB to thank the following individuals for moral support: Prof. Patrick Suppes, Stanford University, Frkan 'Tin, Bilkent University, and Dr. Wlodek Zadrozny, IBM Thomas J. Watson Research Center. Preliminary versions of this paper have been presented as $[1,2,3,4]$. 
the discovery of the Russell's Paradox [6]) throughout its history, which were nevertheless overcome by new axiomatizations.

The most popular of these is the Zermelo-Fraenkel axiomatization with "Choice" (ZFC). ZFC is an elegant theory which inhabits a stable place among other axiomatizations as the mainstream set theory. It provides a "hierarchical" framework. This hierarchy starts with only one abstract entity, the empty sct $(\emptyset)$, forms sets out of previously formed entities cumulatively, and is therefore called the cumulative hierarchy. The coherence of this hierarchy is secured by the Axiom of Foundation (FA) which forbids infinite descending sequences of sets under the membership relation $\in$, such as $\ldots \in a_{2} \in a_{1} \in a_{0} \in a$ (thereby not allowing sets which can be constituents of themselves), and which has sometimes been regarded as a somewhat superficial limitation [6]. Sets which obey the FA are called well-founded sets.

The cumulative hierarchy has provided a precise framework for the formalization of many mathematical concepts [7]. However, it may be asked whether the hierarchy is limiting, in the sense that it might be omitting some sets one would like to have around. Cyclic sets, i.e., sets which can be members of themselves, are examples of such interesting sets which are excluded in ZFC. A set like $a=\{a\}$ is strictly banned in ZFC by the FA since $a$ has no member disjoint from itself. Such sets have infinite descending membership sequences and are called non-well-founded sets. Non-well-founded sets have generally been neglected by the practicing mathematician since the classical well-founded universe was a satisfying domain for his practical concerns. However, non-well-founded sets are useful in modeling various phenomena in computer science, viz. concurrency, databases, artificial intelligence (AI), etc. [8].

McCarthy stressed the feasibility of using set theory in AI and invited researchers to concentrate on the subject in a 1985 specch [9]. Circularity is an often exploited property in various fields of AI, e.g., commonsense reasoning. Rehearsing an example of Perlis [10], if non-profit organizations are considered as individuals, then the organization of all non-profit organizations is a set. It is conceivable that this umbrella organization (called NPO) might want to be a member of itself in order to benefit from having the status of a non-profit organization (e.g., tax exemption). But this implies that NPO must be non-well-founded, i.e., NPO $\in$ NPO.

This paper (also see [11]) investigates an alternative set theory, due to Aczel [12], which uses a graphical representation for sets and thereby allows the representation of non-well-founded sets. A program, called HYPERSOLVER, which can solve systems of equations defined in terms of sets in the universe of this new theory is presented. 


\section{HYPERSET THEORY}

In this section we offer, using [8] and [13], a brief review of the Hyperset Theory, which is an enrichment of the classical ZFC set theory. It is the collection of all the conventional axioms of ZFC modified to be consistent with the new universe involving atoms, except that the FA is now replaced by the AFA (to be explained in the sequel). The sets in this theory are collections of atoms (urclements) or other sets, whose hereditary membership relation can be depicted by graphs. These sets may be well-founded or nonwell-founded, i.e., may have an infinite descending membership sequence, in which case they are also called hypersets.

Sets can be pictured by means of directed graphs in an unambiguous manner. For example, $a=\{b,\{c, d\}\}$ can be pictured by the graph in Figure 1. In this graph, each nonterminal node represents the set which contains the entities represented by the nodes below it. The edges of the graph stand for the hereditary membership relation such that an edge from a node $n$ to a node $m$, denoted by $n \rightarrow m$, means that $m$ is a member of $n$. Since $b, c$, and $d$ are assumed to contain no other entities as elements (i.e., they are urelements), there are no nodes below them.

In Aczel's terminology [12], a pointed graph is a directed graph with a specific node called its point. A pointed graph is said to be accessible if for every node $n$, there exists a path $n_{0} \rightarrow n_{1} \rightarrow \cdots \rightarrow n$ from the point $n_{0}$ to $n$. If this path is always unique, then the pointed graph is a tree and the point is its root. Accessible pointed graphs (apg's) will be used to "picture" sets.

A decoration $D$ for a graph is an assignment of a set to each node of the graph in such a way that

$$
D(n)= \begin{cases}\text { an atom or } \emptyset, & \text { if } n \text { has no children, } \\ \{D(m): n \rightarrow m\}, & \text { otherwise. }\end{cases}
$$

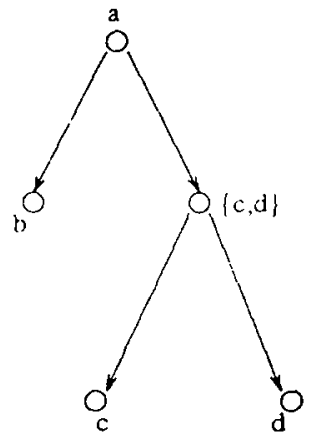

Fig. 1. 'The graph representation of $a=\{b,\{c, d\}\}$. 


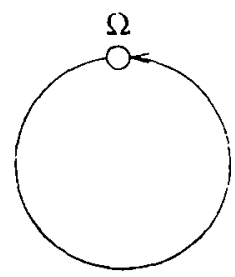

Fig. 2. The picture of the non-well-founded set $\Omega=\{\Omega\}$.

An apg $G$ with point $n$ is a picture of $a$ if there exists a decoration $D(n)=a$, i.e., if $a$ is the set that decorates the top node.

An apg is called well-founded if it has no infinite paths or cycles. Mostowski's Collapsing Lemma states that every well-founded graph has a unique dccoration. As a corollary, every well-founded apg is a picture of a unique well-founded set. A non-well-founded apg can never picture a well-founded set because if $a$ is the set which contains all the sets pictured by the nodes occurring in a cycle of the non-well-founded apg, then it can be seen that no member of $a$ is disjoint from $a$ itself, violating the FA.

Aczel's Anti-Foundation Axiom (AFA) states that every apg, wellfounded or not, pictures a unique set, or stated in other words, every apg has a unique decoration $[12,14]$. The AFA has two implications: existence and uniqueness. The former assures that every apg has a decoration (which leads to the existence of non-well-founded sets besides well-founded ones) and the latter asserts that no apg has more than one decoration. By throwing away the FA from the ZFC (and naming the resulting system $\mathrm{ZFC}^{-}$) and adding the AFA, we obtain the Hyperset Theory (a.k.a. $\mathrm{ZFC}^{-} / \mathrm{AFA}$ ).

One of the important advantages of the new theory is that, by allowing arbitrary graphs, non-well-founded sets are included. For example, the non-well-founded set $\Omega=\{\Omega\}$ is pictured by the apg in Figure 2, and by the uniqueness property of the AFA, this is the only set pictured by that graph. Therefore, there is a unique set which is equal to its own singleton in the universe of hypersets.

The picture of a set can be unfolded into a tree picture of the same set. The tree whose nodcs are the finite paths of the apg which start from the point of the apg, whose edges are pairs of paths $\left\langle n_{0} \rightarrow \cdots \rightarrow n\right.$, $\left.n_{0} \rightarrow \cdots \rightarrow n \rightarrow n^{\prime}\right\rangle$, and whose root is the path $n_{0}$ of length 1 is called the unfolding of that apg. The unfolding of an apg always pictures any set pictured by that apg. Unfolding the apg in Figure 2 results in the infinite tree in Figure 3, analogous to unfolding $\Omega=\{\Omega\}$ to $\Omega=\{\{\{\cdots\}\}\}$.

The uniqueness property of the AFA leads to an intriguing concept of extensionality for hypersets. The classical extensionality paradigm, that 


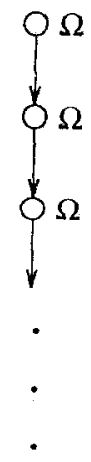

Fig. 3. Unfolding $\Omega$ to obtain an infinite tree.

sets are equal if and only if they have the same members, works fine with well-founded sets. However, this is not of use in deciding the equality of, say, $a=\{1, a\}$ and $b=\{1, b\}$ because it just asserts $a=b$ if and only if $a=b[8]$. However, in the universe of hypersets, $a$ is indeed equal to $b$ since they are depicted by the same graph. ${ }^{1}$

Aczel develops his own extensionality concept by introducing the notion of bisimulation. A bisimulation between two apg's, $G_{1}$ with point $p_{1}$ and $G_{2}$ with point $p_{2}$, is a relation $R \subseteq G_{1} \times G_{2}$ satisfying the conditions

1. $p_{1} R p_{2}$,

2. if $n R m$, then

- for every edge $n \rightarrow n^{\prime}$ of $G_{1}$, there exists an edge $m \rightarrow m^{\prime}$ of $G_{2}$ such that $n^{\prime} R m^{\prime}$

- for every edge $m \rightarrow m^{\prime}$ of $G_{2}$, there exists an edge $n \rightarrow n^{\prime}$ of $G_{1}$ such that $n^{\prime} R m^{\prime}$.

Two apg's $G_{1}$ and $G_{2}$ are said to be bisimilar if a bisimulation exists between them; this means that they picture the same set. It can be concluded that a set is completely determined by any graph which pictures it. Therefore, for two sets to be different, there should be a genuine structural difference between them. For instance, the graphs in Figure 4 all depict the non-well-founded set $\Omega$ because their nodes can be decorated with $\Omega$.

The AFA has interesting applications. In [8], a modeling scheme for propositions (of natural language $[15,16]$ ) is offered. In this scheme, the

\footnotetext{
${ }^{1}$ To see this [8], consider a graph $G$ and a decoration $D$ assigning $a$ to a node $x$ of $G$, i.e., $D(x)=a$. Now consider the decoration $D^{\prime}$ exactly the same as $D$ except that $D^{\prime}(x)=b . D^{\prime}$ must also be a decoration for $G$. But by the uniqueness property of the AFA, $D=D^{\prime}$, so $D(x)=D^{\prime}(x)$, and therefore $a=b$.
} 

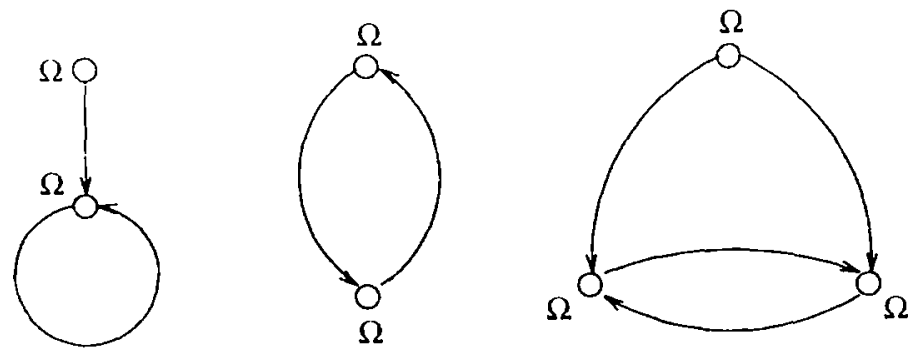

Fig. 4. Other graphs depicting $\Omega$.

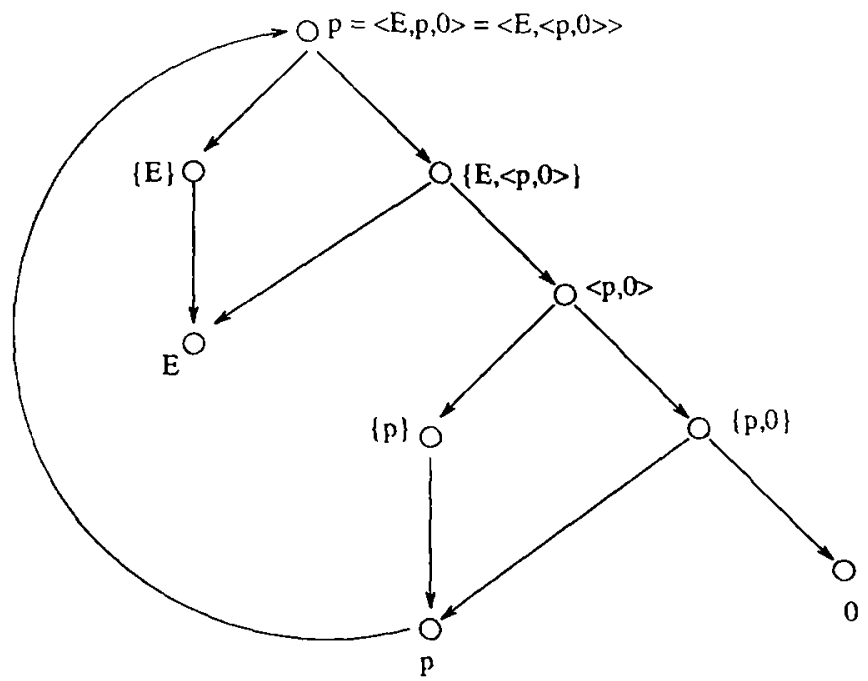

Fig. 5. The picture of "This proposition is not expressible in eight words."

triple $\langle P, p, i\rangle$ denotes that proposition $p$ has property $P$ if $i=1$, and it does not have it if $i=0 .^{2}$ If $p$ is taken to be, say,

"This proposition is not expressible using eight words,"

then it can be modeled by the triple $\langle E, p, 0\rangle$ where $E$ (an atom) denotes the property of being cxpressible (in English) using eight words. In Aczel's conception, $p$ can be depicted as in Figure 5 where the longest arc shows that $p$ refers to itself.

${ }^{2}$ Remember that in set theory, triples such as $\langle x, y, z\rangle$ are defined as pairs of pairs, i.c., $\langle x,\langle y, z\rangle\rangle$, and $\langle y, z\rangle$ is defined as $\{\{y\},\{y, z\}\}$. 


\section{SOLVING SYSTEMS OF HYPERSET EQUATIONS}

The AFA has an important consequence which has useful applications allowing us to assert that some sets exist without having to picture them with graphs. This will be motivated by the following example [12].

An equation of the form $x=\langle 0, x\rangle$ in one variable $x$ can be rewritten as $x=\{\{0\},\{0, x\}\}$. This is equivalent to the following system of four equations in four unknowns:

$$
\begin{aligned}
x & =\{y, z\}, \\
y & =\{w\}, \\
z & =\{w, x\}, \\
w & =0 .
\end{aligned}
$$

By the AFA, this system of equations has a unique solution pictured by the graph in Figure 6 . Unfolding the original equation, one obtains $x=\langle 0,\langle 0,\langle 0, \ldots\rangle\rangle\rangle$.

This result can be generalized. For any set $a$, the equation $x=\langle a, x\rangle$ has a unique solution $x=\langle a,\langle a,\langle a, \ldots\rangle\rangle\rangle$. More generally; an infinite system of equations

$$
\begin{aligned}
& x_{0}=\left\langle a_{0}, x_{1}\right\rangle, \\
& x_{1}=\left\langle a_{1}, x_{2}\right\rangle, \\
& x_{2}=\left\langle a_{2}, x_{3}\right\rangle,
\end{aligned}
$$

has a unique solution

$$
\begin{aligned}
& x_{0}=\left\langle a_{0},\left\langle a_{1},\left\langle a_{2}, \ldots\right\rangle\right\rangle\right\rangle, \\
& x_{1}=\left\langle a_{1},\left\langle a_{2},\left\langle a_{3}, \ldots\right\rangle\right\rangle\right\rangle, \\
& x_{2}=\left\langle a_{2},\left\langle a_{3},\left\langle a_{1}, \ldots\right\rangle\right\rangle\right\rangle,
\end{aligned}
$$

Motivated by such examples, a technique to assert that every system of equations has a unique solution has been developed by Aczel [12]. Named the Solution Lemma by Barwise and Etchemendy [8], this is formulated below.

Let $\mathcal{V}_{A}$ be the universe of hypersets with atoms from a given set $A$. Let $\mathcal{V}_{A^{\prime}}$ be the universe of hypersets with atoms from another given set $A^{\prime}$ such that $A \subseteq A^{\prime}$. Let $X$ be defined as $A^{\prime}-A$. The elements of $X$ can be considered as indeterminates ranging over the universe $\mathcal{V}_{A}$. Sets which can contain atoms from $X$ in their construction are called $X$-sets. A system of 


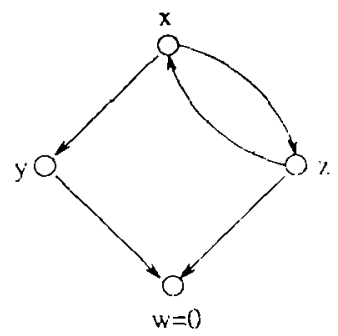

Fig. 6. The solution of the system $x=\{y, z\}, y=\{w\}, z=\{w, x\}, w=0$.

equations is a set of equations.

$$
\left\{x=a_{x}: x \in X \wedge a_{x} \text { is an } X \text {-set }\right\}
$$

for each $x \in X$. For example, choosing $X=\{x, y, z\}$ and $A=\{\mathrm{C}, \mathrm{M}\}$ (thus $A^{\prime}=\{x, y, z, \mathrm{C}, \mathrm{M}\}$ ), we may consider the system of equations

$$
\begin{aligned}
& x=\{\mathrm{C}, y\}, \\
& y=\{\mathrm{C}, z\}, \\
& z=\{\mathrm{M}, x\} .
\end{aligned}
$$

(This system will be used in the sequel for illustrative purposes.)

A solution to a system of equations is a family of pure sets $b_{x}$ (sets which can have only sets but no atoms as elements), one for each $x \in X$, such that for each $x \in X, b_{x}=\pi a_{x}$. Here, $\pi$ is a substitution operation (defined below) and $\pi a$ is the pure set obtained from $a$ by substituting $b_{x}$ for each occurrence of an atom $x$ in the construction of $a$.

The Substitution Lemma states that for each farnily of pure sets $b_{x}(x \in$ $X$ ), there exists a unique operation $\pi$ which assigns a pure set $\pi a$ to each $X$-set, $a$, viz,

$$
\pi a=\{\pi b: b \text { is an } X \text {-set such that } b \in a\} \cup\{\pi x: x \in a \cap X\} .
$$

The Solution Lemma can now be stated [12]. If $a_{x}$ is an $X$-set, then the system of equations $x=a_{x}(x \in X)$ has a unique solution, i.e., a unique family of pure sets $b_{x}$ such that for each $x \in X, b_{x}=\pi a_{x}$.

This lemma can be stated somewhat differently [13]. Letting $X$ again be the set of indeterminates, $g$ a function from $X$ to $2^{X}$, and $h$ a function from $X$ to $A$, there exists a unique function $f$ for all $x \in X$ such that

$$
f(x)=\{f(y): y \in g(x)\} \cup h(x) .
$$


Obviously, $g(x)$ is the set of indeterminates and $h(x)$ is the set of atoms in each $X$-set $a_{x}$ of an equation $x=a_{x}$. In the above example, $g(x)=\{y\}$, $g(y)=z, g(z)=\{x\}$, and $h(x)=\{C\}, h(y)=\{C\}, h(z)=\{11\}$, and one can compute the solution

$$
\begin{aligned}
& f(x)=\{\mathrm{C},\{\mathrm{C},\{\mathrm{M}, x\}\}\}, \\
& f(y)=\{\mathrm{C},\{\mathrm{M},\{\mathrm{C}, y\}\}\}, \\
& f(z)=\{\mathrm{M},\{\mathrm{C},\{\mathrm{C}, z\}\}\},
\end{aligned}
$$

easily. ${ }^{3}$

This technique of solving equations in the universe of hypersets allows us to assert the existence of some sets (the solutions of the equations) without having to depict them with graphs. This feature can be of considerable help in modeling information which can be cast in the form of equations. An example concerning situation theory follows.

Situation theory is a theory of meaning and information content developed by Barwise and Perry [17]. It tries to formalize a semantics for English in the way English speakers handle information. A situation is a limited portion of the reality. An infon is an ordered list $\langle R, a, i\rangle$, where $R$ is a relation, $a$ is a proper sequence of arguments of $R$, and $i$ is the polarity ( 1 or 0 ). For a given $R$ and $a$, only one of the two infons $\sigma=\langle R, a, 1\rangle$ or $\bar{\sigma}=\langle R, a, 0\rangle$ is a fact, namely the one which holds in some situation $s$. (As a notational convention, a polarity of 1 is usually dropped.)

It is gencrally hypothesized that situations are sets of facts and therefore can be inodeled by sets to make use of the existing set-theoretic techniques. Indeed, this was the approach Barwise and Perry adopted in [17]. However, using Barwise's Admissible Set Theory [7] as the principal mathematical tool in the beginning led to problems in the handling of circular situations and they had to turn to the Hyperset Theory $[18,19]$. 'To demonstrate circular situations, an example concerning common knowledge will now be given, viz. the Conway paradox [20]. Two card players $P_{1}$ and $P_{2}$ are given some cards such that each gets an ace. Thus, both $P_{1}$ and $P_{2}$ know that the following is a fact:

$\sigma$ : Either $P_{1}$ or $P_{2}$ has an ace.

${ }^{3}$ The Solution Lemma is an elcgant result, but not every system of equations has a solution. First of all, the equations have to be in the form suitable for the Solution Lemma. For example, a pair of equations $x=\{y, z\}, y=\{1, x\}$ cannot be solved since this requires the solution to be stated in terms of the indeterminate $z$. (Notice the analogy to the Diophantine equations.) As another example, the equation $x=2^{x}$ cannot be solved because Cantor has proved in $\mathrm{ZFC}^{-}$that there is no set which contains its own power set. (no matter what axioms are added to ZFC ${ }^{-}$) [6]. 
When asked whether they knew if the other one had an acc or not, they both would answer "no." If they are told that at least one of them has an ace and asked the above question again, first they both would answer "no." But upon hearing $P_{1}$ answer "no:" $P_{2}$ would know that $P_{1}$ has an ace. Because, if $P_{1}$ does not know $P_{2}$ has an ace, having heard that at least one of them does, it can only be because $P_{1}$ has an ace. Obviously, $P_{1}$ would reason the same way, too. So, they would conclude that each has an ace. Therefore, being told that at least onc of them has an ace must have added some information to the situation. How can being told a fact that each of them already knew increase their information? (This is the Conway paradox.) The solution relies on the observation that initially $\sigma$ was known by each of them, but it was not common knowledge. Only after it became common knowledge did it give more information.

Hence, common knowledge can be viewed as iterated knowledge of $\sigma$ of the following form: $P_{1}$ knows $\sigma, P_{2}$ knows $\sigma, P_{1}$ knows $P_{2}$ knows $\sigma, P_{2}$ knows $P_{1}$ knows $\sigma$, and so on. This iteration can be represented by an infinite sequence of facts (where $\kappa$ is the relation "knows" and $s$ is the situation in which the above game takes place, hence $\sigma \in s$ ):

$$
\left\langle\kappa, P_{1}, s\right\rangle,\left\langle\kappa, P_{2}, s\right\rangle,\left\langle\kappa, P_{1},\left\langle\kappa, P_{2}, s\right\rangle\right\rangle,\left\langle\kappa, P_{2},\left\langle\kappa, P_{1}, s\right\rangle\right\rangle, \ldots
$$

However, considering the system of equations

$$
\begin{aligned}
& x=\left\{\left\langle\kappa, P_{1}, y\right\rangle,\left\langle\kappa, P_{2}, y\right\rangle\right\}, \\
& y=s \cup\left\{\left\langle\kappa, P_{1}, y\right\rangle,\left\langle\kappa, P_{2}, y\right\rangle\right\},
\end{aligned}
$$

the Solution Lcmma asserts the existence of the unique sets $s^{\prime}$ and $s \cup s^{\prime}$ satisfying these equations, respectively, where

$$
s^{\prime}=\left\{\left\langle\kappa, P_{1}, s \cup s^{\prime}\right\rangle,\left\{\kappa, P_{2}, s \cup s^{\prime}\right\rangle\right\} .
$$

Then, the fact that $s$ is common knowledge can be represented by $s^{\prime}$, which contains just two infons and is circular. This is known as the fixedpoint account of common knowledge.

\section{THE IMPLEMENTATION}

HYPERSOLVER is a stand-alone program which can solve equations in the universe of hypersets by making use of the Solution Lemma. It has builtin graphical capabilities for displaying the graphs depicting the equations input by the user and the solutions of these equations. HYPERSOLVER is 


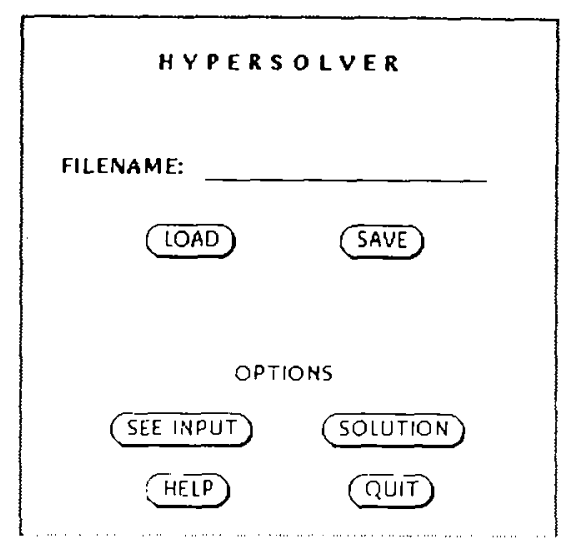

Fig. 7. The command interface of HYPERSOLVER.

implemented on a SPARCstation ELC in Lucid Common Lisp. To communicatc with the user and to display graphs, it makes use of the XView Window Toolkit [21] built on the X Window System. The user interface of HYPERSOLVER, called the command interface, is shown in Figure 7.

\subsection{FUNCTIONALITY}

HYPERSOLVER solves a system of equations in the universe of hypersets. By a system of equations, the definition in Section 3 is meant:

$$
\left\{x=a_{x}: x \in X \text { and } a_{x} \text { is an } X \text {-set }\right\}
$$

for each $x \in X$, where $X$ is a set of indeterminates, $A$ is a set of atoms, and an $X$-set is a set which can contain elements from $X$. HYPERSOLVER does not solve systems which are not of this form. ${ }^{4}$

The notational conventions in HYPERSOLVER are as follows. Let,ters $\mathrm{A}$ through $\mathrm{L}$ are used to represent atoms of $A$, whilc letters $\mathrm{M}$ through $\mathrm{Z}$ represent indeterminates of $X$. The symbol @ will be used to represent the non-well-founded singleton $\Omega$. (One-letter variable naming may seem quite limiting, but it is simple to adopt the parser to handle variables with

${ }^{4}$ Therefore, taking $A=\{0,1\}$ and $X=\{x, y\}$, a system such as $x=\{0,1, y\}$, $y=\{x\}$, is a valid input for HYPERSOLVER, while the single equation $1=\{x, y, 0\}$, or the system $x=\{0,1\}, x=\{x\}$, are not since $1 \notin X$, and there should be a single equation for each $x \in X$. HYPERSOLVER includes some filtering functions to detect invalid input. 


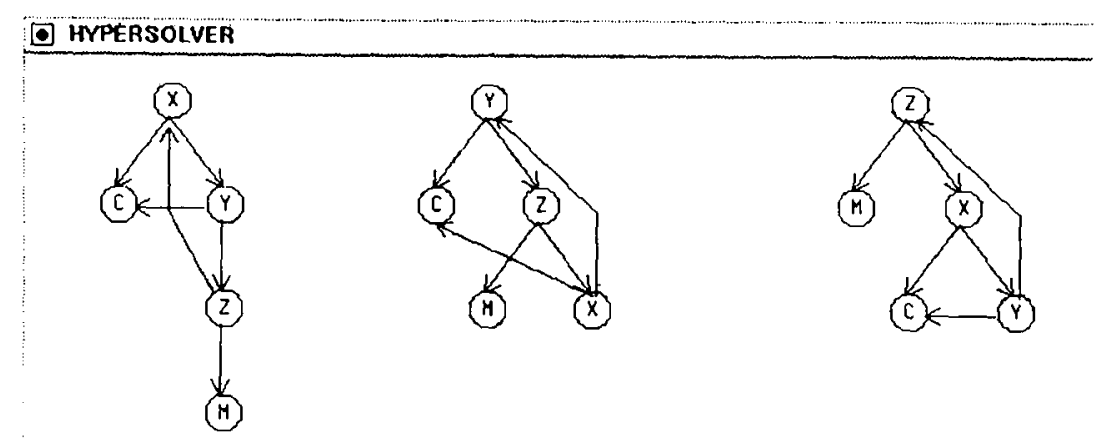

Fig. 8. An example output of HYPERSOLVER.

longer names.) Therefore, the graphs of the solution given in Section 3 are depicted as in Figure 8.

HYPERSOLVER gets its input from a file which is to be specified by the user. The file must have one equation per line. For example, a file consisting of the following lines is a valid input filc:

$$
\begin{aligned}
& X=\{X, Y\}, \\
& Y=\{A, B, Y, Z\}, \\
& Z=\{X, Y, \mathbb{C}\} .
\end{aligned}
$$

The input read from the file is sent to the parser of HYPERSOLVER. The parser is a character-checking parser with a lookup table for the input characters. After converting the input into Lisp form, a transformation is applied to map it to a list that can be processed by the equation solver. Finally, the input is checked to see whether it conforms to the input requirements of HYPERSOLVER (e.g., if it contains one equation for each indeterminate, if each equation is of the form $x=a_{x}$, and so on).

The equation-solving step of the HYPERSOLVER applies the Solution Lemma to the input system of equations. 'The alternative formulation mentioned in Section 3 is used for this purpose:

$$
f(x)=\{f(y): y \in g(x)\} \cup h(x),
$$

for any set $X$ of indeterminates where $g$ is a function from $X$ to $2^{X}$ and $h$ is a function from $X$ to a set $A$ of atoms. For the input file above, $g(\mathrm{X})=\{\mathrm{X}, \mathrm{Y}\}, g(\mathrm{Y})=\{\mathrm{Y}, \mathrm{Z}\}, g(\mathrm{Z})=\{\mathrm{X}, \mathrm{Y}\}$ and $h(\mathrm{X})=\emptyset$, 
$h(\mathrm{Y})=\{\mathrm{A}, \mathrm{B}\}, h(\mathrm{Z})=\{@\}=@$. This representation scheme is suitable for recursive substitution. The algorithm of the equation solver performs this substitution by applying the Substitution Lemma to each equation of the input system. So, the solution for an indeterminate $\mathrm{X}$ can be found by finding the solutions of the indetcrminates in $g(\mathrm{X})$ recursively. For each indeterminate, a decoration is found and the solutions are expressed in terms of thesc decorations. If the decoration for an indeterminate includes itself, then this denotes self-membership, and @ is used to signal that. For example, the decorations of the graphs for the above system of equations are $(p, q$, and $r$ are the decorations for the indeterminates $\mathrm{X}, \mathrm{Y}$, and $\mathrm{Z}$, respectivcly):

$$
\begin{aligned}
& p=\{\mathbb{Q},\{A, B, \mathbb{Q},\{p, q, Q\}\}\}, \\
& q=\{A, B, Q,\{\{\Theta, q\}, q, Q\}\}, \\
& r=\{\{\Theta, q\},\{A, B, Q, r\}, Q\} \text {. }
\end{aligned}
$$

To prevent duplicate substitutions which arise when an indeterminate occurs two or more times in an $X$-set, a list of already visited indeterminates is maintained. Nevertheless, because of the nature of recursion, duplication may occur in different levels of set nesting. Therefore, a kind of filtering is applied on the output of the solver to remove such duplicates.

The next step is the invocation of the graph display part of the HYPERSOLVER. This part takes the solution of a system of equations produced by the equation solver as input. As the general graph layout algorithm, a variant of the hierarchical layout algorithm proposed in [22] is exploited. The reason to use a hierarchical layout algorithm instead of a generalpurpose algorithm is that most of the equations to be solved by the Solution Lemma will be hicrarchical and that self-reference generally occurs for a single indeterminate. (Figure 5 is a good example of this.)

The algorithm which has been adapted to the representation conventions and output requirements of HYPERSOLVER first forms the edge list of the solution system which consists of pairs of nodes. This list helps to get all children of each indeterminate. Then the nodes corresponding to these children are distributed to the levels taking care of the relationships between pairs of nodes. A more complicated part of the graph display unit is the one calculating the positions of the nodes on the screen. The hierarchical nature of the solution graphs is again exploited to make this calculation. The positions of the descendants of a node are calculated with respect to its own position, which in turn has been calculated with respect to its antecedents.

After the calculation of the positions, the actual graph-drawing procedure is activated to display first the nodes and later the edges. This 


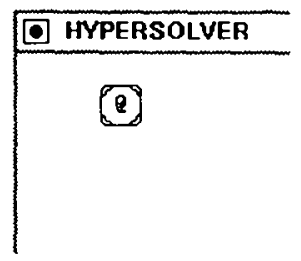

Fig. 9. The HYPERSOLVER graph of $\Omega$.

procedure pops up a large window (called the graph display window, GDW) on which all graphical information is put. The output convention is such that the node labels which are the decorations of the sets represented by those nodes are written inside the node boundarics. Whilc the edges which define hereditary membership are easily drawn, care has to be taken in case of a cycle. Cycles implying self-reference are not displayed as circular edges, but are drawn in a different form. (Therefore, $\Omega$ is depicted as in Figure 9.)

Cycles of one level are not much of a problem. If there exists a cycle between two nodes $a$ and $b$, then the directed edge $(b, a)$ can be drawn over the directed edge $(a, b)$ to give a double arrow. However, references to higher levels, especially to the root node representing the indeterminate, are problematic since a path with minimum edge-crossing has to be found for aesthetic reasons. In such a case, paths walking around the graph are preferred (cf. Figure 12). Edge crossings may be unavoidable if no such path can be found. The solution graphs of the above example are depicted in Figure 10.

The displaying of the graphs depicting the input sets proceeds exactly the same way as the displaying of the solution system. For examplc, the
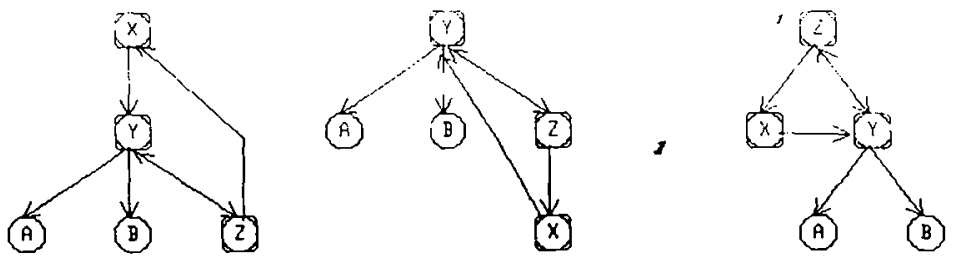

Fig. 10. Graphs depicting the solution to the example in Subsection 4.1. 


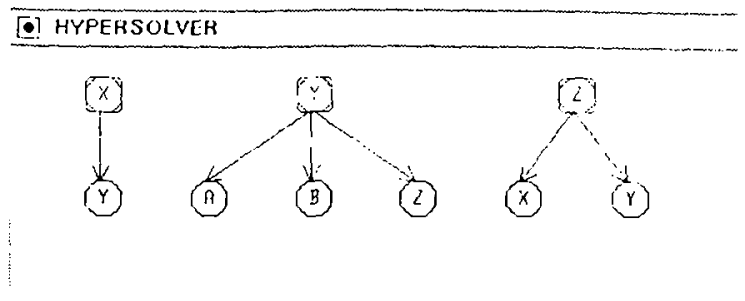

Fig. 11. Graphs of the input equations for the example in Subsection 4.1.

graphs of the input equations of the example system above can be found in Figure 11.

\subsection{LIMITATIONS AND ONGOING WORK}

HYPERSOLVER can solve any system which is in the form required by the Solution Lemma. This requires the equations to be in the form $x=a_{x}$ for each $x \in X$. The systems which cannot be solved by HYPERSOLVER are those to which the Substitution Lemma cannot be applied. Such systems have been exemplified in Section 3 .

HYPERSOLVER is generally weak in input/output operations. First of all, it has limitations on the format of the input, such as one-letter variable naming, and one equation per line in the input filc with no space between the characters of the input equations. These limitations arise because of the brittleness of the parser. A more powerful parser would let HYPERSOLVER be more flexible with input, but the extra features would not add to the power of the program.

The graph display unit is another weak part of HYPERSOLVER. Graph drawing is a hard problem when considered for general graphs with any number of nodes. Limiting the scope of the graph display problem as explained above reduces the difficulties considerably, but classical problems such as minimizing the number of edge-crossings remain. HYPERSOLVER's graph display unit does not claim to know much about the graph layout problem. The algorithm does not work well for arbitrary graphs with no coherent node relationships. However, it works fine for the examples presented so far. Graph-drawing problems are addressed in [23-25], which propose generic graph browsers or editors.

Future work on HYPERSOLVER will concentrate on its applications to modeling of various phenomena in AI. This may include, for example, integrating HYPERSOLVER into a situation-theoretic framework [26] where the program may solve equations whose indeterminates can be unknown 


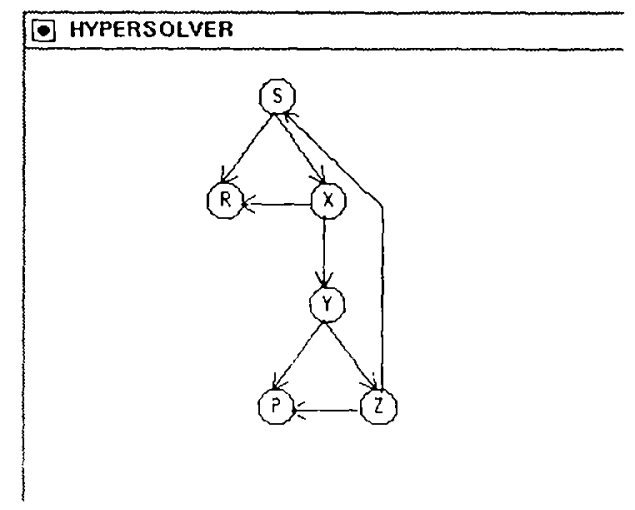

Fig. 12. The graph of a circular situation $S=\langle R, P, S\rangle$. (N.B. Not all the structure is shown.)

elements of situations, or unknown situations themselves. As a simple example, if a situation $S$ is represented by the triple $\left\langle R, P, S^{\prime}\right\rangle$, meaning object $P$ is in relation $R$ to another situation $S^{\prime \prime}$, then $S$ can be found in terms of $S^{\prime}$ by solving the equation $S=\left\langle R, P, S^{\prime}\right\rangle$. Then, if $S$ is a circular situation, $P$ could also be in relation $R$ to $S$ itself, i.e., $S=\langle R, P, S\rangle$. This would, for example, correspond to an actual situation $S$ in which a person $P$ utters the statement "This is a very exciting situation." By "this situation," $P$ is surely referring to the situation which his utterance describes. Such a circular situation $S$ would be depicted (in a somewhat compressed format) as in Figure 12.

HYPERSOLVER's capabilities can also be exploited to model partial information [27]. For this purpose, the objects of the universe $\mathcal{V}_{A}$ (cf. Section 3 ) of hypersets over a set $A$ of atoms can be used to model nonparametric objects, i.e., objects with complete information. The set $X$ of indeterminates can be used to represent parametric objects, i.c., objects with partial information. The universe of hypersets on $A \cup X$ is denoted as $\mathcal{V}_{A}[X]$, analogous to the adjunction of indeterminates in algebra. For any object, $a \in \mathcal{V}_{A}[X]$, the set

$$
\operatorname{par}(a)=\{x \in X: x \in T C(a)\}
$$

where $T C(a)$ denotes the transitive closure of $a$, is called the set of parameters of $a$. If $a^{\prime} \in \mathcal{V}_{A}$, then $\operatorname{par}(a)=\emptyset$, since $a$ does not have any parameters. An anchor is a function $f$ with $\operatorname{domain}(f) \subseteq X$ and $\operatorname{range}(f) \subseteq$ $\mathcal{V}_{A}-A$ which assigns sets to indeterminates. For any $a \in \mathcal{V}_{A}[X]$ and anchor $f, a(f)$ is the object obtained by replacing each indeterminate 
$x \in \operatorname{par}(a) \cap$ domain $(f)$ by the set $f(x)$ in $a$. This can be accomplished by solving the resulting equations using HYPERSOLVER.

\section{CONCLUSION}

The Solution Lernma is a nice feature of the Hyperset Theory. Besides its mathematical importance and elegance, it provides an interesting way of modeling various circular phenomena $[8,28]$.

The implementation presented in this paper, HYPERSOLVER, is a program based on the Solution Lemma and can be a useful tool in areas of AI where information can be cast in the form of set equations. Its simplicity, clarity, and well-defined user interface make it a practical instrument accessible for such purposes. When supported by a more general parser and a better graphical interface, it can be one of the emerging tools in mathematical logic, along the lines of, e.g., Suppes and Sheehan's computerized set theory course [29], or Barwise and Etchemendy's Tarski's World [30].

HYPERSOLVER may be an important utility for basic research on the use of set theory in AI, too [31]. Such research involving conceptual innovations is urgently nceded in AI, as pointed out by McCarthy [32].

\section{REFERENCES}

1. V. Akman, Set theory from Zermelo to Aczel. Seminar, Department of Mathematics, Bilkent University, Ankara, March 10, 1992.

2. V. Akman, Hypersets and common sense. Seminar, Department of Computer Engineering, Middle East Technical University, Ankara, October 21, 1992.

3. M. Pakkan, Solving equations in the universe of hypersets. Master's thesis, Department of Computer Enginecring and Information Science, Bilkent University, Ankara, Turkey, 1993.

4. M. Pakkan and V. Akman. HYPERSOLVER: A graphical tool for commonsense set. theory. In Proceedings of the Fighth International Symposium on Computer and Information Sciences (ISCIS VIII), L. Gün, R. Önvural, and E. Gelenbe, Eds., İstanbul, 1993, pp. 436-443.

5. J. R. Shoenfield, Axioms of set theory. In Handbook of Mathernatical Logic, J. Barwise, ed., North-Holland, Amsterdam, 1977, pp. 321-344.

6. A. A. Fraenkel, Y. Bar-Hillel, and A. Levy, Foundations of Set Theory. NorthHolland, Amsterdarn, 1973.

7. J. Barwise, Admissible Sets and Structures. Springer-Verlag, Berlin, 1975.

8. J. Barwise and J. Etchemendy, The Liar: An Essay on Truth and Circularity. Oxford University Press, NY, 1987.

9. J. McCarthy, Acceptance address of the International Joint Conference on Artificial Intelligence (IJCA I-85) award for research excellence, Los Angeles, C $\Lambda$, 1985.

10. D. Perlis, Commonsense set theory. In Meta-Level Architectures and Reflection, P. Maes and D. Nardi, Eds., Flsevier (North-IIolland), Amsterdam, 1988, pp. 87- 98 . 
11. M. Pakkan and V. Akman, Issues in commonsense set theory. In Proceedings of the First Turkish Symposium on Artificial Intelligence and Neural Networks, K. Oflazer, V. Akman, and U. Halici, Eds., Bilkent University, Arkara, 1992, pp. 47-52.

12. P. Aczel, Non-well-founded sets. Number 14 in CSLI Lecture Notes. Center for the Study of Language and Information, Stanford, CA, 1988.

13. J. Barwise and L. Moss, Hypersets, Mathematical Intelligencer 13(1):31-41 (1991).

14. J. Barwise, AFA and the unification of information. In The Situation in Logic, number 17 in CSLI Lecture Notes, Center for the Study of Language and Information, Stanford, CA, 1989, pp. 277-283.

15. C. McLarty, Anti-foundation and self-reference. Journal of Philosophical Logic 22:19-28 (1993).

16. D. Perlis, Languages with self-reference I: Foundations. Artificial Intelligence 25(3):301-322 (1985)

17. J. Barwise and J. Perry, Situations and Attitudes. MIT Press, Cambridge, MA, 1983.

18. J. Barwise, Situations, sets, and the axiom of foundation. In The Situation in Logic, number 17 in CSLI Lecture Notes, Center for the Study of Language and Information, Stanford, CA, 1989, pp. 177-200.

19. J. Barwise, Situated set theory. In The Situation in Logic, Center for the Study of Language and Information, Stanford, CA, 1989, Chap. 14.

20. J. Barwise, On the model theory of common knowledge. In The Situation in Logic, number 1.7 in CSLI Lecture Notes, Center for the Study of Language and Information, Stanford, CA, 1989, pp. 201-220.

21. D. Heller, XVieu Programming Manual. O'Reilly \& Associates, Sebastopol, CA, 1990 .

22. L. A. Rowe, M. Davis, F. Messinger, C. Meyer, C. Spirakis, and A. Tuan, A browser for directed graphs. Software-Practice and Experience 17(1):61-76 (1987).

23. E. R. Gansner, S. C. North, and K. P. Vo, DAG- $\Lambda$ program that draws directed graphs. Software-Practice and Experience 18(11):1047-1062 (1988).

24. T. Kamada and S. Kawai, An algorithm for drawing general undirected graphs. Information Processing Letters 31:7-15 (1989).

25. F. N. Paulisch and W. F. Tichy, EDGE: An extensible graph editor. Software-Practice and Experience 20(S1):63-88 (1990).

26. E. Tin and V. Akman, BABY-SIT: Towards a situation-theoretic computational environment. In Current Issues in Mathematical Linguistics, C. Martin-Vide, Ed., Elsevier Science B.V., Amsterdam, 1994, pp. 299-308.

27. M. W. Mislove, L. S. Moss, and F. J. Oles, Partial sets. In Situation Theory and its Applications I, R. Copper, K. Mukai, and J. Perry, Eds., number 22 in CSII Lecture Notes, Center for the Study of Language and Information, Stanford, CA, 1990, pp. 117-131.

28. L. S. Moss, Review of The Liar: An Essay on Truth and Circularity. Bulletin of the American Mathematical Society 20(2):216-225 (1989).

29. P. Suppes and J. Sheehan, CAI course in axiomatic set theory. In University-level Computer-Assisted Instmuction at Stanford: 1968-1980, P. Suppes, Ed., Institute for Mathematical Studies in the Social Sciences, Stanford University, Stanford, CA, 1981, pp. 3-80.

30. J. Barwise and J. Etchemendy, The Language of First-Order Logic (including the Macintosh version of Tarski's World 4.0), 3rd ed. Number 23 in CSLI Lecture Notes. Center for the Study of Language and Information, Stanford, CA, 1993. 
31. W. Zadrozny, Cardinalities and well orderings in a common-sense set theory. In Proceedings of the First International Conference on Principles of Knowledge Representation and Reasoning, R. J. Brachman, H. J. Levesque, and R. Reiter, Eds. Morgan Kaufmann, San Mateo, CA, 1989, pp. 486-497.

32. J. McCarthy, Artificial intelligence needs more emphasis on basic research: President's quarterly message. AI Magazine 4:5 (1983).

Received 1 February 1993; revised 30 October 1994 